\title{
Time-sensitive effects of Zhuang Medicated Thread Moxibustion on Estrogen Level in female perimenopausal Model Rats.
}

\author{
Dan Liang ${ }^{1}$, Yun Zhang ${ }^{1}$, Zhiyong $\mathrm{Cao}^{2}$, Jingqing Chen ${ }^{1}$, Zhuli Hou ${ }^{2}$, Yuhan $\mathrm{Pan}^{1}$, Ke Lu${ }^{1}$, \\ and Gang Fang ${ }^{1}$ \\ ${ }^{1}$ GuangXi University of Chinese Medicine \\ ${ }^{2}$ Hubei Minzu University
}

April 9, 2021

\begin{abstract}
Abstract Background: To ascertain the estrogenic effect of Zhuang Medicated Thread Moxibustion (ZMTM) and explore its time-sensitive impact on estradiol in female perimenopausal rats. Methods: Female rats were randomized into four groups of 10 rats, each consisting of the control, model, ZMTM, and acupuncture groups. The perimenopausal syndrome was induced in the last three groups with a daily subcutaneous dose of $80 \mathrm{mg} / \mathrm{kg}$ of 4- vinylcyclohexene diepoxide for 15 days. Afterward, rats in the model and control groups were fed routinely, while rats in the ZMTM and acupuncture groups were treated with six ZMTM and acupuncture courses, respectively. The rats' general condition and estradiol (E2) levels in the rats' serum were assessed. Results: Following the six courses of treatment, the E2 level in the model group was significantly the lowest, while the regular group was the highest $(\mathrm{P}<0.05)$. There was also a gradual increase in the E2 level of the ZMTM group compared to the model and acupuncture groups, such that after the 5th and 6th courses of treatment, their E2 level was significantly higher than the model and acupuncture groups $(\mathrm{P}<0.05)$. The control group had a better condition than all other groups, while the ZMTM group was better than the model and acupuncture groups. Conclusion: ZMTM can improve perimenopausal induced rats' estrogen level, and this effect becomes better with each further treatment course.
\end{abstract}

Time-sensitive effects of Zhuang Medicated Thread Moxibustion on Estrogen Level in female perimenopausal Model Rats.

\section{Abstract}

Background: To ascertain the estrogenic effect of Zhuang Medicated Thread Moxibustion (ZMTM) and explore its time-sensitive impact on estradiol in female perimenopausal rats.

Methods: Female rats were randomized into four groups of 10 rats, each consisting of the control, model, ZMTM, and acupuncture groups. The perimenopausal syndrome was induced in the last three groups with a daily subcutaneous dose of $80 \mathrm{mg} / \mathrm{kg}$ of 4- vinylcyclohexene diepoxide for 15 days. Afterward, rats in the model and control groups were fed routinely, while rats in the ZMTM and acupuncture groups were treated with six ZMTM and acupuncture courses, respectively. The rats' general condition and estradiol $\left(\mathrm{E}_{2}\right)$ levels in the rats' serum were assessed.

Results: Following the six courses of treatment, the $\mathrm{E}_{2}$ level in the model group was significantly the lowest, while the regular group was the highest $(\mathrm{P}<0.05)$. There was also a gradual increase in the $\mathrm{E}_{2}$ level of the ZMTM group compared to the model and acupuncture groups, such that after the $5^{\text {th }}$ and $6^{\text {th }}$ courses of treatment, their $\mathrm{E}_{2}$ level was significantly higher than the model and acupuncture groups $(\mathrm{P}<0.05)$. The control group had a better condition than all other groups, while the ZMTM group was better than the model and acupuncture groups. 
Conclusion: ZMTM can improve perimenopausal induced rats' estrogen level, and this effect becomes better with each further treatment course.

Keywords : ZMTM, perimenopausal syndrome, estrogen, time-sensitiveness, Chinese Medicine, Experimental.

What is already known about this topic?

Perimenopausal syndrome (PPS) is also known as climacteric syndrome, whose main symptoms include endocrine dyscrasia and neurasthenia induced by the degeneration of ovarian functions ${ }^{2}$. It is also known as a perimenopausal disorder in traditional Chinese medicine. The primary treatment is hormone replacement therapy, which can relieve climacteric symptoms and negative emotions, but it has side effects ${ }^{4}$. Other research shows that long-term hormone therapy increases breast cancer risk, endometrial cancer, ovarian cancer, and other related diseases ${ }^{4}$.

What does this article add?

This study confirms that Zhuang Medicated Thread Moxibustion can improve perimenopausal model rats' estrogen levels; this curative effect is most effective at the 3rd cycle and tends to become better in further treatment cycles. Although the mechanism of action of ZMTM is yet to be explored, making further human trials necessary, this study has however, laid a foundation for its clinical research and application.

\section{BACKGROUND}

Perimenopausal syndrome (PPS) is also known as climacteric syndrome, whose main symptoms include endocrine dyscrasia and neurasthenia induced by the degeneration of ovarian functions ${ }^{2}$. It is also known as a perimenopausal disorder in traditional Chinese medicine. As a disease in women, PPS mainly occurs around the time of menopause. According to a study by Lijuan et.a.l ${ }^{3}$, the probability of having PPS in women is the highest (92.10\%) among ages 45 to 55 years, and PPS seriously affects their quality of life and living standard ${ }^{3}$. The perimenopausal syndrome is a disease with high morbidity. It can have severe physical and psychological effects on perimenopausal women. According to modern medicine, the diseases' significant causes include the central nervous system and the autonomic nervous system. The primary treatment is hormone replacement therapy, which can relieve climacteric symptoms and negative emotions, but it has side effects ${ }^{4}$. Other research shows that long-term hormone therapy increases breast cancer risk, endometrial cancer, ovarian cancer, and other related diseases ${ }^{4}$. Therefore, the exploration of new treatments is vital for improving the quality of life of women.

Estrogen is of great significance in the regular operation of the female endocrine system ${ }^{5}$. Receptors respond to estrogen for estrogen to exert its effect. It is interesting to note that estrogen's primary receptor, ER- $\alpha$, is closely linked to neuron growth, function, and structure. Contemporary literature shows that estradiol (E2)'s positive influence on gonadotropin secretion occurs primarily in the hypothalamus; however, it negatively influences the pituitary gland ${ }^{6}$. The leading causes of PPS include low estrogen levels and degeneration of ovarian function. Therefore, an increase in estrogen levels provides a foundation for treating PPS.

Zhuang Medicated Thread Moxibustion (ZMTM) is a characteristic surgical procedure established on the theory of "yin-yang basis", the man-nature theory featured by the synchrony of triple initial energy, and the theory of "Sandao lianglu" which is concerned with the tract for the digestion and absorption of food, tract of water, tract of energy, tract of blood, and sensing tract ${ }^{1}$.

The procedure for ZMTM entails a ramie thread (No. 2 thread with a diameter of $70 \mathrm{~mm}$ ), which is first soaked in Chinese herbs and then ignited and applied on the patient's special acupoints to stimulate those acupoints through heat and medicine. Therefore, energy can be regulated after treatment on the body surface (or acupoints) and the blood and the sensing tract ${ }^{1}$.

According to the literature, the expression of specific factors can be regulated, and cell apoptosis improved through $\mathrm{ZMTM}^{7,8}$. When ovariectomized rabbits are treated with ZMTM, the interleukin-2 (IL-2) level in their serum can be increased. The high rates of apoptosis in splenocytes can be improved, their immunity 
can be enhanced, and the negative influence on the uterus shape and ER- $\alpha$ expressions improved ${ }^{7,8}$. Based on previous studies' results, this study was designed from the perspective of time-sensitive effects of ZMTM on the level of estrogen in perimenopausal model rats. Therefore, the aim is to investigate the influence of ZMTM on the changes of estrogen in PPS and related features, which can thus provide a reference and favorable implication for clinical application.

\section{Methods}

This prospective and randomized study was conducted at the Dept. of Medicine of Hubei Minzu University and Guangxi University of Chinese Medicine, performed for two years from September 2018 to September 2020. The Institutional Review Board and the local ethical committee have approved the study after registration with the Research Center (protocol Gui Ke AD1924518). This study followed the criteria as declared by the ARRIVE guidelines 2.0.

\section{Experimental animals}

Female Sprague-Dawley rats (40 in number) $(180-220 \mathrm{~g})$ about six weeks old were acquired from the Changsha Topgene Biotechnology Co., Ltd; (certification: SCXK (Xiang) 2014-001. The animals had been housed under managed conditions with a $22 \pm 2 \mathrm{degC}$ controlled temperature, $50 \%+-5 \%$ air humidity, 12 $\mathrm{h}$ light $-12 \mathrm{~h}$ dark cycle, and fed routinely with access to tap water ad libitum and allowed to acclimatize for one week before the commencement of the experimental study. The maintenance and treatment of rats was carried out strictly following the Care and Use of Laboratory Animals' guidelines published by the U.S. National Academies Press (Eighth Edition, update, 2011). The committee approved the experimental protocols on the Ethics of Animal Experiments of the School of Zhuang Medicine, Guangxi University of Chinese Medicine.

Randomization of consecutive rats was done using the slot method, and the rats were divided into four groups: the standard control group $(n=10)$, the model group $(\mathrm{n}=10)$, the acupuncture group $(n=10)$, and ZMTM group $(\mathrm{n}=10)$. A single operator performed all procedures on the groups' rats under standard aseptic conditions and protocols to remove bias. The First operator and second operator (readings) were double-blind. And a third operator was used for evaluating the data.

Zhuang medicated thread moxibustion, alcohol lamp, glass slides, normal saline, $1 \mathrm{ml}$ syringe, and cotton swabs were all provided by the School of Zhuang Medicine laboratory, Guangxi University of Chinese Medicine. 4-vinyl cyclohexene dioxide (4-VCD), Rat $\mathrm{E}_{2}$ ELISA KIT (Shanghai Meilian Biotech Co., Ltd.), inverted research microscope (DM18, Leica, Germany), multifunctional microplate reader (M200 TECAN, Switzerland), and high-speed freezing centrifuge (3K15 Sigma, Germany).

The standard control and model groups received no treatment. They were routinely fed and had access to water. However, for rats in the ZMTM group, they received ZMTM. After the thread was ignited and the acupoints were located, the operator used his wrist and fingers to press the thread's ignited tip on the acupoints swiftly. When the glow was extinguished, the whole operation could be seen as one "Zhuang ." Typically, each acupoint was acupunctured for one "Zhuang." Again, the no.2 thread was selected, which was $70 \mathrm{~mm}$ in diameter, and when the ignited thread was held over the acupoints, a $1-2 \mathrm{~cm}$ tail of the thread should be left intact. Disinfection of acupoints was not necessary before applying ZMTM because of the heat. Typical acupoints include Xiaguanyuan, Qizhousi acupoints, and Beibaacupoints, which are characteristic in Zhuang medicine, mainly for treating miscellaneous gynecological diseases ${ }^{9}$. Acupoints on rats were located according to Experimental Acupuncture Science ${ }^{10}$. The ZMTM was applied to acupoints once per day, 2 Zhuang per acupoint, for six courses. Rats in the acupuncture group were treated with 0.28 filiform needles with the same acupoints, and the treatment time was similar to the ZMTM group. Vaginal smears collection time was strictly based on the menstrual cycle of female rats. Typically, one course of treatment lasts for one menstrual cycle.

\section{Model preparation and evaluation}

The model, acupuncture, and ZMTM groups received a daily subcutaneous injection of 4 -VCD at $80 \mathrm{mg} / \mathrm{kg}$ 
for 15 consecutive days. The $200 \mathrm{~g}$ rats were given a volume of $0.2 \mathrm{ml}(80 \mathrm{mg} / \mathrm{ml})$, i.e., $0.001 \mathrm{ml} / \mathrm{g}$, for 15 consecutive days to induce the perimenopausal model. After five days of treatment, rat vaginal smears were collected from each group for five consecutive days. The estrous cycle of rats was confirmed by microscopic evaluation of cells in vaginal smears. In all three groups, besides the standard control group, rat vaginal smears showed estrous symptoms, i.e., a significant amount of leukocytes with some epithelial cells and keratinocytes were present, which implies that the perimenopausal model was induced successfully. No mortality was seen during the induction of the perimenopausal phase.

\section{Sample collection}

$2 \mathrm{ml}$ venous blood from each rat's eye socket was collected in the 1st, 3rd, 5 th, and 6th courses of treatment and was kept in estrogen receptor tubes at $4 \mathrm{deg} \mathrm{C}$. When blood coagulation occurred, the tubes were placed in a centrifuge. After centrifugation, the supernatant was collected and kept in a freezer at -20degC. Rat vaginal smears were collected simultaneously for $\mathrm{H} \& \mathrm{E}$ staining, which was done in Guangxi National Hospital and observed by the microscope. All rats were sacrificed by cervical dislocation under anesthesia.

\section{Measurement of serum Estradiol $\left(\mathbf{E}_{2}\right)$}

ELISA kit was used, and the standard operation was completed according to instructions.

\section{Statistical analysis}

SPSS 19.0 was the software used for analyzing results. Data calculated as mean +- S.D.; standard deviation ( $+-\mathrm{s})$. Intragroup data were compared using a one-factor analysis of variance (ANOVA). The differences are statistically significant when the p-value is less than 0.05 .

\section{RESULTS}

$\mathrm{H} \& \mathrm{E}$ staining of rat vaginal smears from each group is shown in Figure 1

\section{Comparing the general condition of rats in each group after six courses of treatment}

: The mental state, disposition, feed intake, reaction, and general well-being of all rats among all groups were examined at the end of the treatment course. The standard control rats were generally stable, mentally agile, and had a regular feed intake and disposition. On the contrary, the model group rats were restless, with slow reaction, inadequate feed intake, and physically weak. However, the treatment group, i.e., ZMTM, showed the improved $(\mathrm{p}<0.05)$ mental status of the rats and feed intake. Although the rats' disposition was docile, there was a general improvement in the condition of the rats. The results obtained for the ZMTM group appear similar to those of the acupuncture group. Table 1

\section{The effect of ZMTM on $\mathrm{E}_{2}$ of perimenopausal model rats}

The results of E 2 levels are shown in Figures 2 and 3. As seen from Figure 2, the standard control group had the highest estradiol level from cycle one all through to process 6 . However, the model group had significantly $(\mathrm{p}<0.05)$ lower $\mathrm{E}_{2}$ values, which was the lowest among all groups, and this was maintained low from cycle one all through to cycle six. It was interesting to observe that as treatment with ZMTM commenced from cycle 1 , a significant increase $(\mathrm{p}<0.05)$ in $\mathrm{E} 2$ levels was noted. This increase was steadily improved significantly $(\mathrm{p}<0.05)$ from cycle one through to cycle six, with the increase in E2 levels being highest on the $6^{\text {th }}$ cycle. The effect of ZMTM on estrogen levels was similar to the acupuncture group on the $3^{\text {rd }}$ cycle but significantly higher $(\mathrm{p}<0.05)$ on the $5^{\text {th }}$ and $6^{\text {th }}$ cycles.

Figure 3 gives an insight into the $\mathrm{E}_{2}$ level in the sixth cycle. It can be seen here that the ZMTM treatment significantly $(\mathrm{p}<0.05)$ improved the level of E2 compared to the perimenopausal model group and the E2 value seen for the ZMTM group was comparable to the standard control group and better than the acupuncture group. This leads to the reversal of the perimenopausal effect as induced by 4 -VCD.

\section{DISCUSSION}


A perimenopausal syndrome is a common female disease typified by neurasthenia and neuropsychological symptoms, mainly caused by an alteration in the hormone levels around menopause ${ }^{11}$. According to traditional Chinese medicine, PPS falls into the category of hysteria and perimenopausal disorder. The leading causes include deficiency of kidney qi, stagnation of liver qi, internal disturbance of seven emotions, and stress, injuring qi and blood. There are many therapeutic approaches to handling PPS. According to research, standard acupuncture and electro-acupuncture can relieve the symptoms of menopause ${ }^{12}$. According to Zhuang medicine, "heiq" (qi) and "lwed" (blood) play an essential role in female growth and development. When women are in perimenopause, it means the degeneration of their physical quality and body function. The decline in "heiq" (qi) and "lwed" (blood) will cause the disorder of qi and blood and finally lead to PPS. As a traditional external therapy, ZMTM can regulate qi and blood through acupoints to balance qi and blood, relieve symptoms, improve body health, and finally show good therapeutic efficacy ${ }^{9}$. Essential therapy tools include medicated thread made from many Zhuang herbs, e.g., Santalum album, musk, frankincense, and Argyreia acuta. It has a delicate smell and can achieve good therapeutic effects ${ }^{13}$. Qizhousi acupoints, Beiba acupoints, and other selected acupoints are characteristic acupoints in Zhuang medicine, mainly treating various gynecological diseases.

Perimenopausal syndrome correlates with changes in sex hormones. Therefore, the difference in sex hormones should be given close attention during treatment by the treating physicians. Furthermore, the physicians should examine the levels of luteinizing hormone (L.H.), stimulating follicle hormone (FSH), estradiol (E2), and other hormones to assist in the diagnosis and treatment of $\mathrm{PPS}^{14}$. This study indicates that rat estrogen levels decreased after perimenopause model induction and improved after ZMTM treatment. This effect of ZMTM was significant right from the 1 st to the $6^{\text {th }}$ cycle. However, its influence became notable from the $3^{\text {rd }}$ cycle and was most meaningful and useful between the 5th and 6th cycles. $\mathrm{E}_{2}$ levels in the ZMTM group were significantly higher $(\mathrm{p}<0.05)$ than the acupuncture group and model group, although slightly lower than the standard group.

\section{Conclusion}

This study confirms that ZMTM can improve perimenopausal model rats' estrogen levels; this curative effect is most effective at the 3rd cycle and tends to become better in further treatment cycles. Although the mechanism of action of ZMTM is yet to be explored, making further human trials necessary, this study has however, laid a foundation for its clinical research and application.

\section{Abbreviations}

ZMTM: Zhuang Medicated Threads; PPS: Perimenopausal Syndrome; E: Estradiaol; IL-2: Interleukin 2.

Ethics approval and consent to participate: All animal experiments were authorized by the Guangxi Zhuang Yao Medicine Center of Engineering and Technology, Guangxi University of Chinese Medicine and Hubei Minzu University Animal Ethics Committee and carried out in compliance with the institutional guidelines.

Consent to publish : Not Applicable

Availability of data and materials : The datasets used and/or analyzed during the current study are available from the corresponding author on reasonable request.

Competing interests: The authors declare that they have no competing interests

Funding : This work was sponsored by the National Natural Science Foundation of China (No.81660830) ;Guangxi Program on Science and Technology Project: Gui Ke AD1924518;

Development Program of High-level Talent Team under Qihuang Project of Guangxi University of Chinese Medicine(No.2018005);Guangxi first class discipline construction project (No. Gui Jiao Ke Yan [2018]12). The funders had no role in study design, data collection and analysis, decision to publish, or preparation of manuscript. 
Authors' Contributions : K.L. is mainly responsible for the design and operation of the whole experiment. Y.Z., Z.C., and J.C. primarily assisted in the process of the investigation. Z.H. and Y.P. wrote and revised the manuscript. DL and G.F. provided the overall idea for the experiment and revised the paper in the later stage. All authors have read and approved the manuscript before submission.

Acknowledgements : Not Applicable

\section{REFERENCES}

1. Yaoping J, Jiang L, Gaofeng Ma, Jing X, Jiyong M. Effect of Zhuang Medicated Thread Moxibustion on Synovial cell apoptosis in rats with adjuvant arthritis. Lishizhen Med Materia Medica Res 2017; 28(8): 2028-2031.

2. Ye L, Mingfang Z, Junwei W. Clinical study of Kunbao pills in the treatment of Perimenopausal syndrome. Lishizhen Med Materia Medica Res 2013; 24(8): 1957-1958.

3. Lijuan H, Jing Z, Yanli Y. Clinical observation on treatment of perimenopausal syndrome with Zishen Tiaogan Jianpi decoction. Ningxia Medical J 2018; 40 (4): 371-373.

4. Li L, Niaz M, Yanping B, Youlin Q. Retrospective analysis of the relationship between hormone replacement therapy and gynecological tumors in perimenopausal women. Chin J Practical Gynecol Obste 2010; 26(11): 849-852.

5. Chaohui T. Efficacy of Bushen huayu decoction combined with estrogen in the treatment of perimenopausal syndrome with kidney deficiency and blood stasis. Lishizhen Med Materia Medica Res 2017; 28(4): 915-916.

6. Lindzey Jonathan, Jayes Friederike L, Yates Mariana M, Couse John F, Korach Kenneth S. The bimodal effects of estradiol on gonadotropin synthesis and secretion in female mice are dependent on estrogen receptor-alpha. J Endoerinol, 2006; 191(1): 309-317.

7. Meichun Y, Xinxin M, Jing L, Lijuan H, Gang F. Effect of Zhuang Medicated Thread Moxibustion on apotosis of spleen cells and expression of uterine ER- $\alpha$ in ovariectomized rabbits. Lishizhen Med Materia Medica Res 2012; 10(4): 254-255.

8. Gang F, Jing L, Lijuan H, Xiaqing X, Meichun Y. Effects of Zhuang Medicated Thread Moxibustion on serum interleukin-2 in ovariectomized rabbits. Hebei Trad Chinese Med 2011; 33(04): 592-594.

9. Jinming H, Chen L. Zhuang Medicated Thread Moxibustion. Nanning: Guangxi Nationalities Publishing House, 2006; 10.

10. Zhongren L. Experimental Acupuncture and Moxibustion. Beijing: China Press of Traditional Chinese Medicine, Appendix (Atlas of Rat Acupoints), 2002.

11. Junwei T, Qin L, Yidan M, Lijuan B. Treatment of perimenopausal syndrome with integrated Chinese and Western medicine (Case clinical study). Jiangsu Trad Chin Med 2019; 51(1): 45-47.

12. Xuechua Y, Lirong Y, Xinjing S. Research progress of acupuncture therapy for perimenopausal syndrome. Journal of Hebei North University (Natural science edition) 2018; 34(12): 55-60.

13. Meichun Y, Lijuan H, Jing L, Gang F, Xiaqing X. The effect of Zhuang Medicated Thread Moxibustion on sex hormones in ovariectomized rabbits. Chinese Acupuncture Moxibustion 2011; 31(2): 145-148.

14. Caihua Z. The effect of low dose estrogen replacement therapy in perimenopausal syndrome. J Gansu Sci Technol 2019, 35(1): 132-133.

\section{Figure Legends:}

Figure 1: H \& E staining of rat vaginal smears from each group (100x) and Vaginal smears of rats in estrous cycle.

A: Pre estrus: a great number of nucleated epithelial cells and some cornified epithelial cells are present.

B: Estrus period: all are cornified cells without cell nucleus, with some epithelial cells present.

C: Late estrus: mostly cornified cells and leukocytes are present.

D: Estrus interval: most leukocytes and some mucosa and epithelial cells are present. 
Figure 2: Time-sensitiveness of $\mathrm{E}_{2}$ in rats after perimenopausal model induction

Figure 3: Comparison of $\mathrm{E}_{2}$ levels in rats after perimenopausal model induction on the sixth cycle

Figure 1
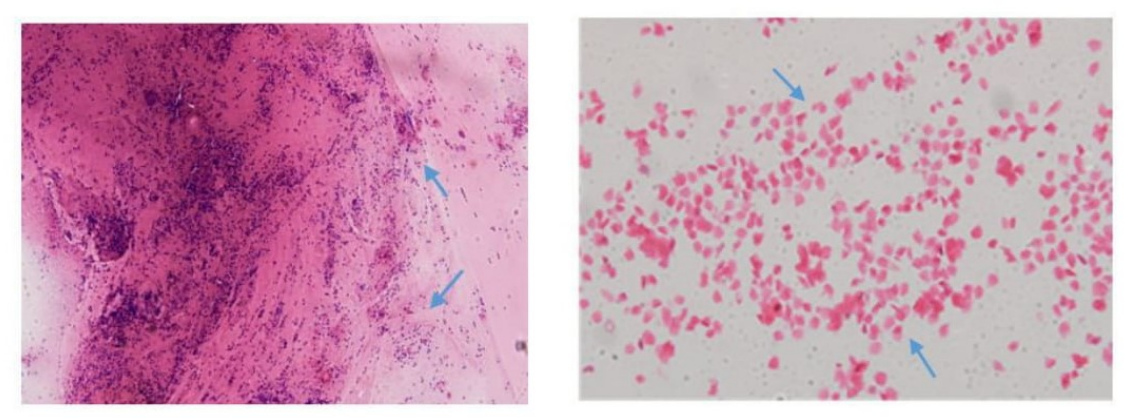

A

B
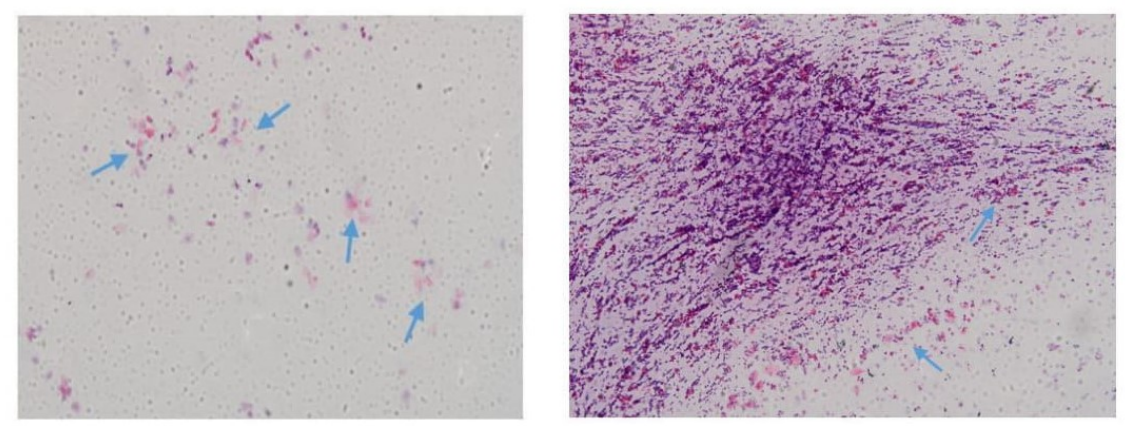

$\mathrm{C}$

$\mathrm{D}$ 
Figure 2

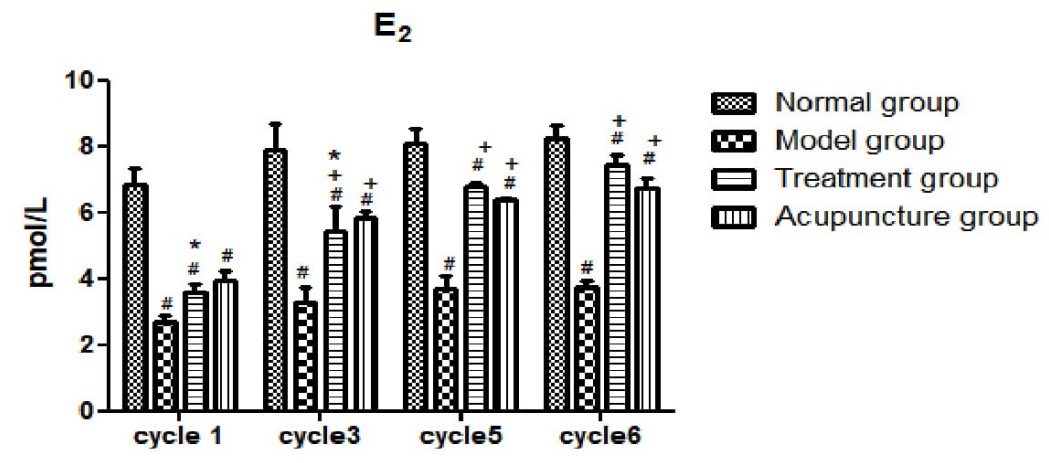

Note: ${ }^{\#} \mathrm{P}<0.05$ compared with the normal group, ${ }^{+} \mathrm{P}<0.05$ compared with the model group,

${ }^{*} \mathrm{P}>0.05$ compared with the control group.

Figure 3

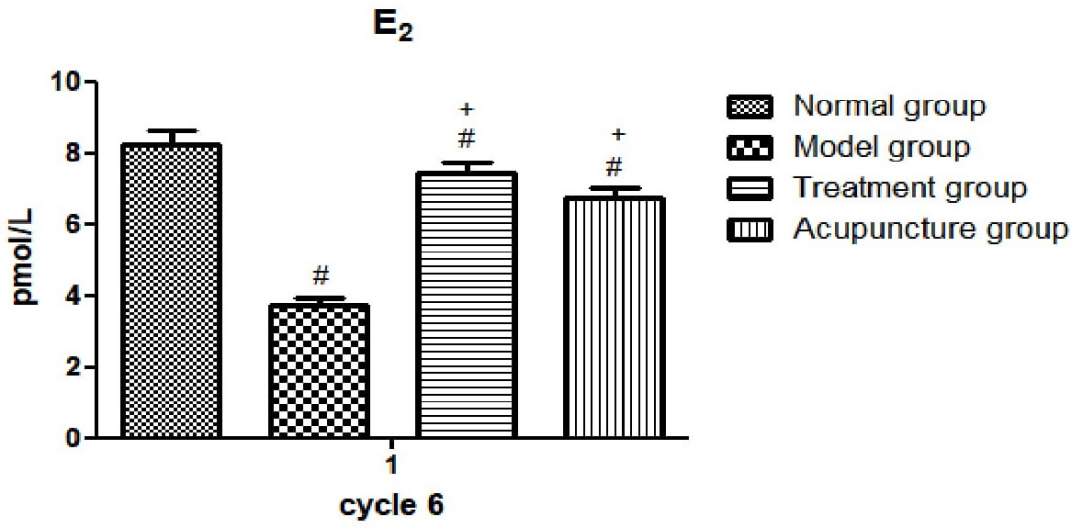

Note: ${ }^{\#} \mathrm{P}<0.05$ compared with the normal group, ${ }^{+} \mathrm{P}<0.05$ compared with the model group,

${ }^{*} \mathrm{P}>0.05$ compared with the control group.

\section{Hosted file}

Table 1.pdf available at https://authorea.com/users/406804/articles/517333-timesensitive-effects-of-zhuang-medicated-thread-moxibustion-on-estrogen-level-in-femaleperimenopausal-model-rats 